\title{
Imprinting disorder in donor cells is detrimental to the development of cloned embryos in pigs
}

\author{
Xuexiong Song ${ }^{1, *}$, Fangzheng $\mathrm{Li}^{1, *}$, Zhongling Jiang ${ }^{1, *}$, Yueping Sun ${ }^{1}$, Huatao $\mathrm{Li}^{1}$, \\ Shansong Gao' ${ }^{1}$,iping Zhang ${ }^{1}$, Binghua $\mathrm{Xue}^{2}$, Guimin Zhao ${ }^{3}$, Jingyu Lí2 ${ }^{2}$ Zhonghua \\ Liu ${ }^{2}$, Hongbin $\mathrm{He}^{3}$ and Yanjun Huan ${ }^{1}$ \\ ${ }^{1}$ College of Veterinary Medicine, Qingdao Agricultural University, Qingdao, Shandong Province, China \\ ${ }^{2}$ College of Life Science, Northeast Agricultural University, Harbin, Heilongjiang Province, China \\ ${ }^{3}$ College of Life Science, Shandong Normal University, Jinan, Shandong Province, China \\ *These authors contributed equally to this work
}

Correspondence to: Yanjun Huan, email: huanyanjun1982@163.com

Keywords: imprinting, donor cell, somatic cell nuclear transfer, cloned embryo, pig

Received: June 20, $2017 \quad$ Accepted: August 06, $2017 \quad$ Published: August 22, 2017

Copyright: Song et al. This is an open-access article distributed under the terms of the Creative Commons Attribution License 3.0 (CC BY $3.0)$, which permits unrestricted use, distribution, and reproduction in any medium, provided the original author and source are credited.

\section{ABSTRACT}

Imprinting disorder during somatic cell nuclear transfer usually leads to the abnormality of cloned animals and low cloning efficiency. However, little is known about the role of donor cell imprinting in the development of cloned embryos. Here, we demonstrated that the imprinting (H19/Igf2) in porcine fetus fibroblasts derived from the morphologically abnormal cloned fetuses (the abnormal imprinting group) was more hypomethylated, and accordingly, significantly higher H19 transcription and lower Igf 2 expression occurred in comparison with those in fibroblasts derived from morphologically normal cloned fetuses (the normal imprinting group) or donor fetus fibroblasts (the control group). When these fibroblasts were used as donor cells, the abnormal imprinting group displayed an even lower imprinting methylation level, in correspondence to the significantly downregulated expression of Dnmt1, Dnmt3a and Zfp57, and a markedly reduced blastocyst rate, while the normal imprinting group took on the similar patterns of imprinting, gene expression and embryo development to the control group. When 5 -aza-dC was applied to reduce the fibroblasts imprinting methylation level in the normal imprinting group, cloned embryos displayed the more severely impaired imprinting and significantly lower blastocyst rate. While the upregulated $\mathrm{H19}$ transcription in the abnormal imprinting group was knocked down, the imprinting statuses were partly rescued, and the cleavage and blastocyst rates significantly increased in cloned embryos. In all, donor cell imprinting disorder reduced the developmental efficiency of cloned embryos. This work provides a new insight into understanding the molecular mechanism of donor cells regulating the cloned embryo development.

\section{INTRODUCTION}

Somatic cell nuclear transfer (SCNT) has achieved in many species, owning a broad application prospect in the basic research, agriculture, biomedicine, etc [1]. However, the overall cloning efficiency remains low, and the developmental abnormalities frequently occur, limiting the wide application of cloning technology [2, 3].
It is generally believed that the developmental abnormalities of cloned animals and low cloning efficiency are largely due to the imprinting disorder [4]. Imprinting is an epigenetic regulatory mechanism to ensure a monoallelic parental-specific expression pattern and the normal growth and development of embryos [5]. Then, the imprinting disorder would alter the expression patterns of imprinted genes, resulting in the poor embryo 
development. Thus, imprinting has been considered as an excellent model to evaluate the developmental efficiency of cloned embryos.

Naturally, genomic imprinting is erased and established during gametogenesis and faithfully maintained throughout the subsequent embryo development in the normal reproduction [5]. Indeed, during early embryogenesis, genomic imprinting is recognized and protected by the specific DNA binding complexes including Dnmt1, Zfp57 and Trim28, et al., to resist the global DNA demethylation and remethylation [6]. As for animal cloning, SCNT bypasses the progress of imprinting erasure and establishment, seeming that just the imprinting maintenance could support the cloned embryo development, however, cloned embryos suffers imprinting defects [7], indicating that the imprinting maintenance mechanism is destroyed in cloned embryos, and SCNT may establish or maintain the wrong imprinting, resulting in the poor cloning efficiency.

At present, H19/Igf2, representing genomic imprinting, is widely studied and critical for the normal embryo development [4]. Igf2 paternally expresses and acts as a growth factor, while the transcription of H19, a long noncoding RNA, is maternal. The parent-specific expression of H19/Igf2 is controlled by the differentially methylated region 3 (DMR3, widely accepted) of H19 imprinting control region (ICR). The DMR3 is methylated on the paternal allele, then the enhancer element prefers Igf2 paternal expression. On the maternal allele, H19 transcription has a cis silencing effect on the adjacent Igf2 expression. This mechanism allows for the precise control of $\mathrm{H} 19$ and Igf2 expression [8]. During the assisted reproduction, H19/Igf2 imprinting hypomethylation usually occurs, and the upregulated H19 transcription leads to the developmental defects [9]. As for the semi-cloning, H19 DMR deletion in androgenetic haploid embryonic stem cells can efficiently support the fullterm development of semi-cloned embryos [10]. And, the developmental failure of uniparental embryos also reveals the indispensable role of gametic H19/Igf2 imprinting in the normal embryo development [11]. Then, it is wondered whether the H19/Igf2 imprinting in donor cells, just like in gametes, regulates the cloned embryo development. As the hypomethylated H19/Igf2 imprinting usually occurs during SCNT, and the methylation status of donor cell lines can also affect the cloned embryo development [12-14], then, it is speculated that a close relationship between H19/Igf2 imprinting status in donor cells and the cloned embryo development must exist, needing to be clarified.

Numerous studies have demonstrated that H19/ Igf2 imprinting problems constrain the cloning efficiency [15-17], and our previous studies also revealed that the retarded development of cloned embryos and fetuses was associated with the H19/Igf2 imprinting disorder, and alteration of donor cell DNA methylation impairs the cloned embryo development could be due to the disrupted H19/Igf2 imprinting in donor cells [4, 18]. Thus, in this study, the role of donor cell imprinting in the development of cloned embryos was investigated. Our results demonstrated that when fibroblasts derived from the morphologically abnormal cloned fetus with the hypomethylated H19/Igf2 imprinting were used as donor cells, cloned embryos displayed a markedly reduced development and an even more severely impaired H19/Igf2 imprinting, and downregulation of H19/Igf2 methylation level in normal imprinting PFFs by 5-aza$\mathrm{dC}$ also resulted in the significantly lower blastocyst rate. While H19 transcription was knocked down in abnormal imprinting group, H19/Igf2 imprinting status and the cloned embryo development were obviously improved. This work would have important implications in improving the cloning efficiency.

\section{RESULTS}

\section{Disrupted imprinting in fibroblasts derived from the morphologically abnormal cloned fetuses}

Our previous study has shown that 4 of 6 porcine cloned fetuses were morphologically abnormal and their imprinting levels were hypomethylated [4]. Here, the methylation statuses and transcription of H19/Igf2 and the expression of genes related to the imprinting methylation maintenance were further detected in PFFs (porcine fetus fibroblasts) derived from the morphologically abnormal and normal cloned fetuses (Figure 1 and Supplementary Figures A1, A2, A3, A4). The results demonstrated that the imprinting of PFFs (Supplementary Figures A1, A2, A3, A4) derived from the morphologically abnormal cloned fetuses (A1, A2, A3 and A4) was hypomethylated $(20.42 \%, 27.08 \%, 33.33 \%$ and $35.00 \%$, respectively), and accordingly, the significantly upregulated H19 transcription and downregulated Igf2 expression occurred in comparison with those of PFFs (Supplementary Figures N1 and N2) derived from the morphologically normal cloned fetuses (Supplementary Figures N1 and N2) and donor PFFs. And more, the expression levels of Dnmt1 and Zfp57 also significantly decreased in PFFs (Supplementary Figures A1, A2, A3, A4). While no significant differences of H19/Igf2 imprinting and the expression of Dnmt1 and Zfp57 were observed between PFFs (Supplementary Figures N1 and N2) and donor PFFs. Thus, imprinting was disrupted in fibroblasts derived from the morphologically abnormal cloned fetuses.

\section{Imprinting disorder in donor cells reduced the developmental efficiency of cloned embryos}

To investigate whether donor cell imprinting plays a critical role in the development of cloned embryos, PFFs a2 (not a1, due to the poor cell proliferation, Supplementary Figure 2A and Supplementary Figure 3), representing the abnormal imprinting group, PFFs n2, as 
the normal imprinting group, and donor PFFs, namely the control group, were employed. Here, compared with the control group, the abnormal imprinting group displayed an even lower imprinting methylation level (15.63\% vs $21.88 \%$ in 4 -cell embryos and $18.75 \%$ vs $27.60 \%$ in blastocysts, respectively, Figure $2 \mathrm{~A}$ ), the significantly higher H19 transcription at the 4-cell stage but interestingly and obviously lower H19 expression in blastocysts and the significantly reduced expression of Igf2, Dnmt1, Dnmt3a and Zfp57 in cloned embryos (Figure 2B), and a markedly reduced blastocyst rate was also observed $(12.24 \%$ vs $20.08 \%, P<0.05$, Figure $2 \mathrm{C}$ and

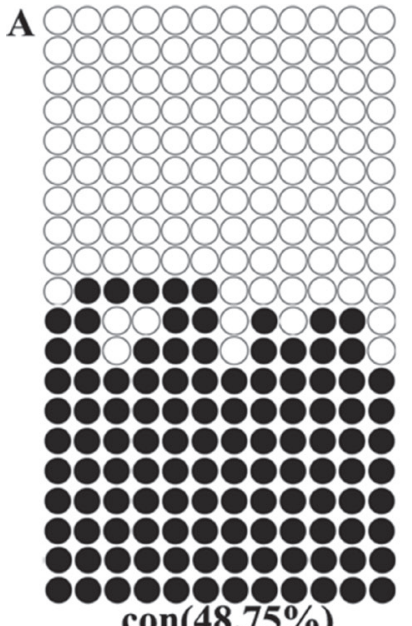

con $(\mathbf{4 8 . 7 5 \% )}$

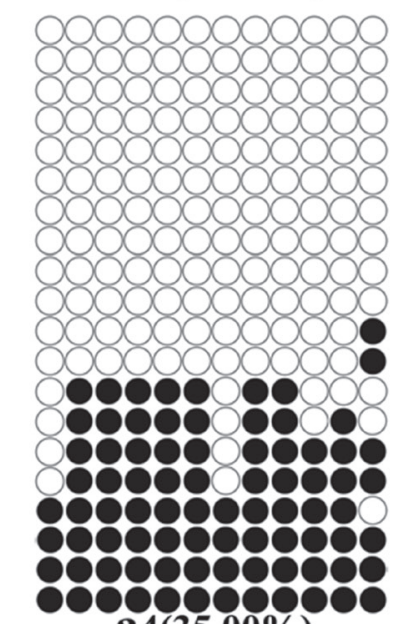

C a4(35.00\%)

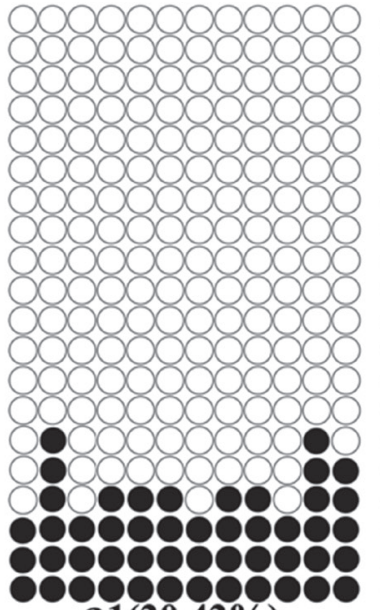

a1 $(20.42 \%)$

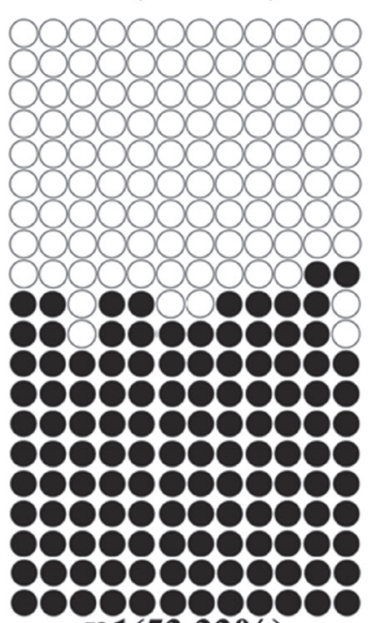

n1 $(53.33 \%)$

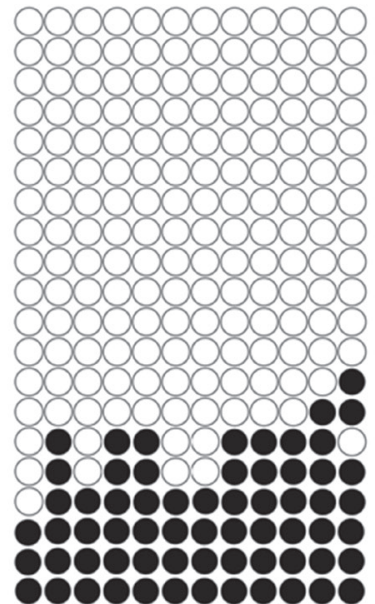

a2(27.08\%)

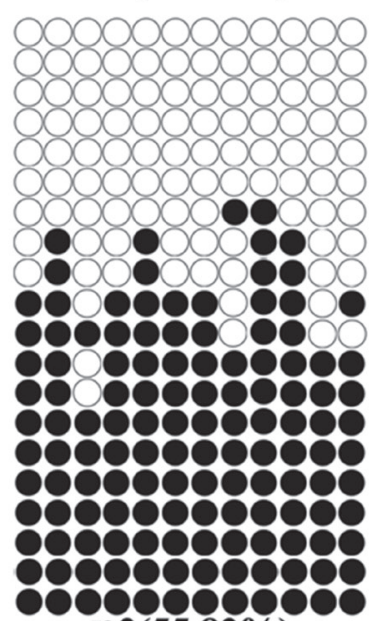

n2(55.83\%)

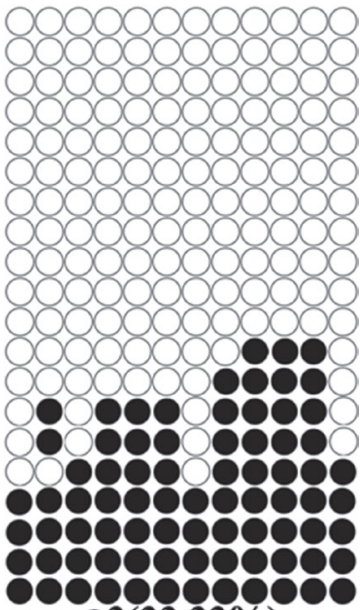

a3(33.33\%)

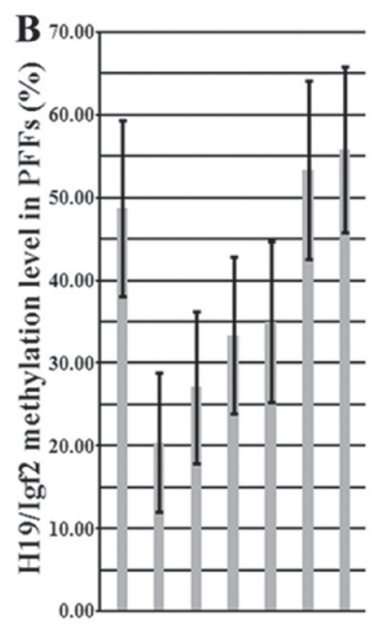

con a1 a2 a3 a4 n1 n2

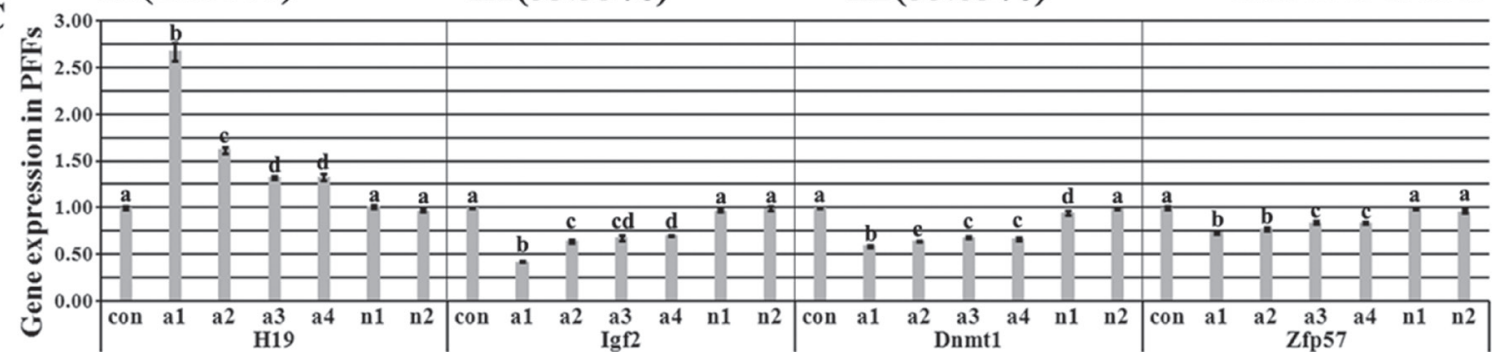

Figure 1: The methylation statuses and transcription of H19/Igf2 imprinting and the expression of genes related to the imprinting methylation maintenance in PFFs. (A) the methylation statuses of H19/Igf2 imprinting in PFFs derived from in vivo fertilized and cloned fetuses, (B) H19/Igf2 methylation levels in PFFs derived from in vivo fertilized and cloned fetuses, (C) the transcription of H19/Igf2 and genes related to the imprinting methylation maintenance in PFFs derived from in vivo fertilized and cloned fetuses. PFFs derived from the morphologically abnormal cloned fetuses displayed the hypomethylated H19/Igf2 imprinting, upregulated H19 transcription and downregulated expression of Igf2 and imprinting methylation maintenance related genes. con represented PFFs derived from the in vivo fertilized fetuses, a1, a2, a3 and a4 represented PFFs derived from the morphologically abnormal cloned fetuses, and $\mathrm{n} 1$ and $\mathrm{n} 2$ represented PFFs derived from the morphologically normal cloned fetuses, respectively. Black or white circles indicate methylated or unmethylated CpG sites, respectively. ${ }^{a-d}$ Values for a given gene with different superscripts differ significantly $(P<0.05)$. 
Table 1: Development of cloned embryos derived from donor cells with the various imprinting statuses

\begin{tabular}{lccccc}
\hline \multicolumn{1}{c}{ Group } & $\begin{array}{c}\text { No. } \\
\text { embryos } \\
\text { (Rep.) }\end{array}$ & $\begin{array}{c}\text { No. embryos fused } \\
(\% \pm \text { SEM) }\end{array}$ & $\begin{array}{c}\text { No. embryos } \\
\text { cleaved }(\% \pm \text { SEM) }\end{array}$ & $\begin{array}{c}\text { No. blastocysts } \\
(\% \pm \text { SEM) }\end{array}$ & $\begin{array}{c}\text { Blastocyst cell numbers } \\
(\text { mean } \pm \text { SEM) }\end{array}$ \\
\hline Control & $221(5)$ & $174(78.79 \pm 2.35)$ & $151(86.94 \pm 1.34)$ & $35(20.08 \pm 0.95)^{\mathrm{a}}$ & $36 \pm 2(n=34)$ \\
$\begin{array}{l}\text { Abnormal } \\
\text { imprinting }\end{array}$ & $225(5)$ & $163(72.55 \pm 1.61)$ & $133(81.62 \pm 2.07)$ & $20(12.24 \pm 0.85)^{\mathrm{b}}$ & $34 \pm 2(n=17)$ \\
$\begin{array}{l}\text { Normal } \\
\text { imprinting }\end{array}$ & $227(5)$ & $175(77.04 \pm 1.93)$ & $149(85.36 \pm 2.02)$ & $40(22.75 \pm 1.14)^{\mathrm{a}}$ & $37 \pm 3(n=39)$ \\
\hline
\end{tabular}

${ }^{\#}$ Cleavage and blastocyst rates were adjusted for fusion rates.

\&Blastocyst cell numbers, less than 16 , were not included.

${ }^{a-b}$ Values in the same column with different superscripts differ significantly $(P<0.05)$.

Table 1). For the normal imprinting group, the imprinting status, gene expression and embryo development were similar to those in the control group (Figure 2). Taken together, these results suggested that serial nuclear transfer did not improve the cloning efficiency and imprinting disorder in donor cells was detrimental to the cloned embryo development.

\section{Donor cell imprinting hypomethylation induced by 5-aza-dC led to the poor developmental efficiency of cloned embryos}

To investigate whether imprinting hypomethylation in donor cells was the cause of the poor cloned embryo development in the abnormal imprinting group, 5-aza$\mathrm{dC}$ was employed to reduce the donor cell imprinting methylation level in the normal imprinting group, then, the imprinting statuses, the expression patterns of genes related to the imprinting methylation maintenance and the cloned embryo development were examined. After PFFs were treated with 5-aza-dC, the obviously downregulated imprinting methylation levels were observed (36.46\% vs $52.08 \%$ at 72 h, $25.52 \%$ vs $50.52 \%$ at $96 \mathrm{~h}$, and $22.40 \%$ vs $51.56 \%$ at $120 \mathrm{~h}$, respectively, Figure 3A) in comparison with those in PFFs untreated, and treating PFFs for $96 \mathrm{~h}$ did not markedly affect the cell proliferation (Supplementary Figure 2B) and was further applied in the Aza (+) group. When these treated PFFs were used as donor cells, compared with those in the Aza (-) group, the greatly lower imprinting methylation levels $(13.54 \%$ vs $22.92 \%$ in 4 -cell embryos and $16.67 \%$ vs $30.21 \%$ in blastocysts, respectively, Figure 3B), the significantly upregulated $\mathrm{H} 19$ transcription in 4-cell embryos but downregulated H19 expression in blastocysts (Figure 3C), the markedly reduced expression of Igf2, Dnmt1, Dnmt3a and Zfp57 in cloned embryos (Figure 3C) and the significantly lower blastocyst rate $(15.40 \%$ vs $22.95 \%, P<0.05$, Figure $3 \mathrm{D}$ and Table 2$)$ occurred in the Aza (+) group. Thus, imprinting hypomethylation in donor cells could be detrimental to the cloned embryo development.

\section{H19 knockdown in the abnormal imprinting donor cells was beneficial for the cloned embryo development}

To further investigate whether the upregulated H19 transcription in donor cells resulted in the poor cloned embryo development, siRNA was employed to reduce H19 expression in the abnormal imprinting group. When siRNA was transfected into PFFs a2, no significant decrease of cell number during PFFs culture occurred (Supplementary Figure 2C), and H19 transcription was significantly knocked down (59.00\%, 32.41\%, 17.19\%, $18.88 \%, 23.54 \%$ or $25.19 \%$ at 6 h, 12 h, 24 h, 36 h, 48 h or $72 \mathrm{~h}$ in the siRNA-positive group vs $100.02 \%$ at $6 \mathrm{~h}$ in the siRNA-control group, respectively, $P<0.05$, Figure 4A). Then, donor cells with H19 knockdown for $24 \mathrm{~h}$ were used for SCNT, and the siRNA-positive group took on the upregulated imprinting methylation levels $(20.31 \%$ vs $14.06 \%$ or $15.10 \%$ in 4 -cell embryos and $31.77 \%$ vs $19.27 \%$ or $18.23 \%$ in blastocysts, respectively, Figure 4B) in comparison with the siRNA-control or siRNA-negative group, suggesting that H19 knockdown in donor cells could rescue the impaired imprinting in cloned embryos. Responding to the ameliorated imprinting, the significantly downregulated H19 transcription in 4-cell embryos and the upregulated Igf2 expression in cloned embryos were observed, and the expression levels of Dnmt1, Dnmt3a and Zfp57 in blastocysts were significantly higher in the siRNA-positive group compared with the siRNAcontrol or siRNA-negative group $(P<0.05$, Figure 4C). Interesting, the significantly higher H19 transcription in blastocysts was also observed in the siRNA-positive group, seemingly inconsistent with its imprinting methylation status. Notably, compared with the siRNAcontrol or siRNA-negative group, the siRNA-positive group displayed the significantly upregulated cleavage and blastocyst rates $(87.28 \%$ vs $78.82 \%$ or $79.32 \%$ for the cleavage rate, and $27.90 \%$ vs $12.85 \%$ or $11.34 \%$ for 
the blastocyst rate, respectively, $P<0.05$, Figure 4D and Table 3). Thus, H19 knockdown in abnormal imprinting donor cells was beneficial for the cloned embryo development.

\section{DISCUSSION}

It is known that abnormal imprinting results in the low cloning efficiency [19]. In this study, our results displayed that the imprinting status in PFFs derived from the morphologically abnormal cloned fetuses was disordered, and these PFFs led to the severely impaired imprinting in cloned embryos and the markedly downregulated blastocyst rate. The poor development of cloned embryos derived from PFFs with the hypomethylated imprinting induced by 5 -aza-dC also demonstrated that the imprinting disruption in donor cells reduced the development of cloned embryos. Additionally, the result that H19 knockdown in donor cells with the hypomethylated imprinting improved the cloned embryo development further supported that donor cell imprinting status could determine the cloning efficiency. Thus, the developmental efficiency of cloned embryos was closely associated with the donor cell imprinting status.

Generally, imprinting disorder results in the developmental abnormalities of cloned animals and low cloning efficiency [4]. Here, we demonstrated that the imprinting status in PFFs derived from the
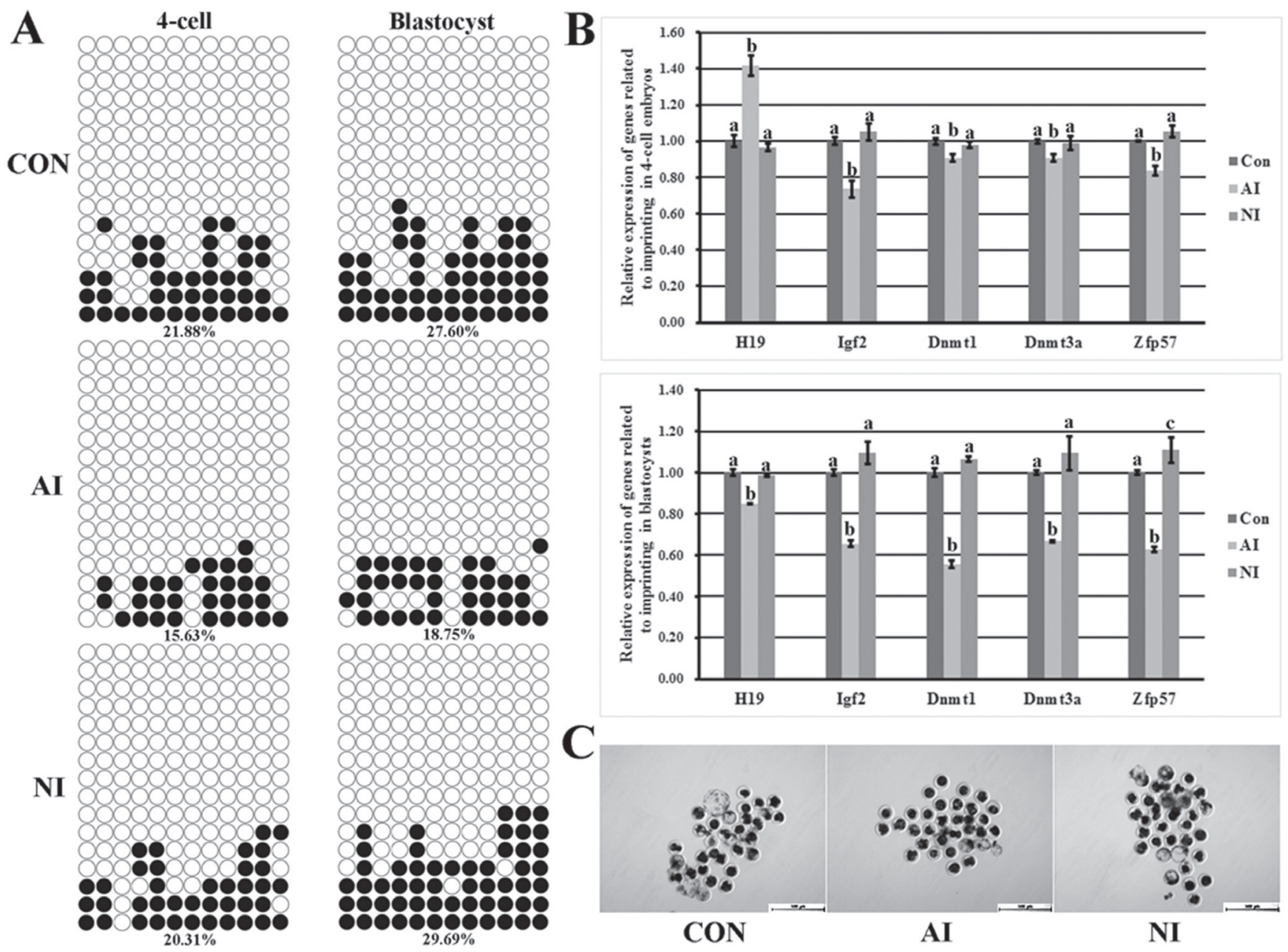

Figure 2: The methylation statuses and transcription of H19/Igf2 imprinting and the expression of genes regulating imprinting methylation in cloned embryos and the cloned blastocysts derived from the control, abnormal imprinting and normal imprinting PFFs. (A) the methylation statuses of H19/Igf2 imprinting at the 4-cell and blastocyst stages of cloned embryos derived from the control, abnormal imprinting and normal imprinting PFFs, (B) the transcription of H19/Igf2 and genes regulating imprinting methylation at the 4-cell and blastocyst stages of cloned embryos derived from the control, abnormal imprinting and normal imprinting PFFs, (C) the cloned blastocysts derived from the control, abnormal imprinting and normal imprinting PFFs (Scale bar $=500 \mu \mathrm{m})$. The cloned embryos derived from the abnormal imprinting PFFs displayed the hypomethylated H19/Igf2 imprinting, disrupted H19 transcription, reduced expression of Igf 2 and genes regulating imprinting methylation and downregulated blastocyst rate. CON, AI and NI represented cloned embryos derived from the control, abnormal imprinting and normal imprinting PFFs, respectively. Black or white circles indicate methylated or unmethylated $\mathrm{CpG}$ sites, respectively. ${ }^{\mathrm{a}-\mathrm{c}}$ Values for a given gene with different superscripts differ significantly $(P<0.05)$. 
Table 2: Development of cloned embryos derived from the normal imprinting donor cells treated with 5-aza-dC

\begin{tabular}{lccc}
\hline Group & No. embryos (Rep.) & No. embryos cleaved (\% \pm SEM) & No. blastocysts $(\% \pm$ SEM) \\
\hline Aza $(-)$ & $135(5)$ & $118(87.76 \pm 1.39)$ & $28(22.95 \pm 1.96)^{\mathrm{a}}$ \\
Aza $(+)$ & $157(5)$ & $127(82.19 \pm 1.65)$ & $20(15.40 \pm 0.61)^{\mathrm{b}}$ \\
\hline
\end{tabular}

${ }^{a-b}$ Values in the same column with different superscripts differ significantly $(P<0.05)$.

morphologically abnormal cloned fetuses was disrupted, coinciding with the previous report [20]. Notably, along with the smaller sizes of the abnormal cloned fetuses, the even lower methylation levels and more disrupted expression patterns of imprinting genes were observed. Whereas, no obvious differences were observed between the morphologically normal cloned group and the in vivo fertilized group. Collectively, these results further

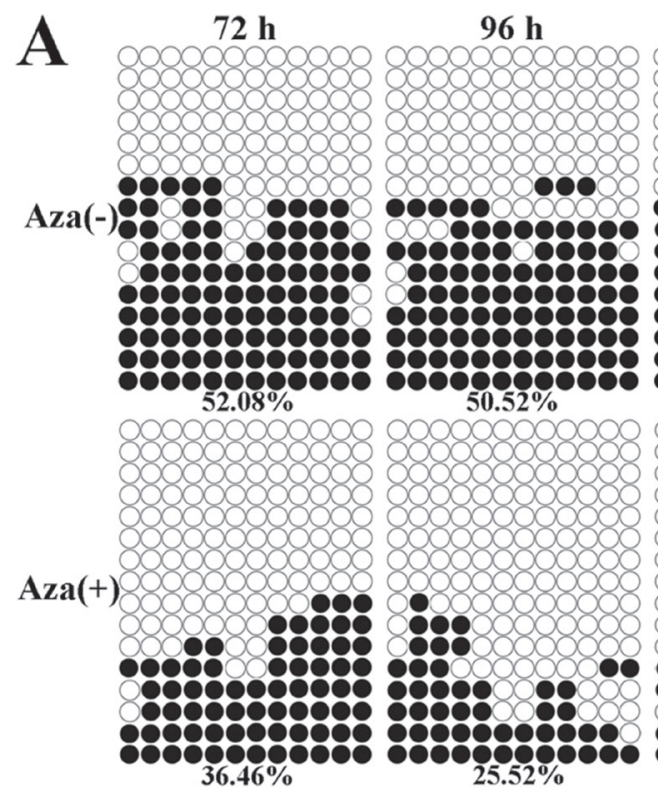

$120 \mathrm{~h}$

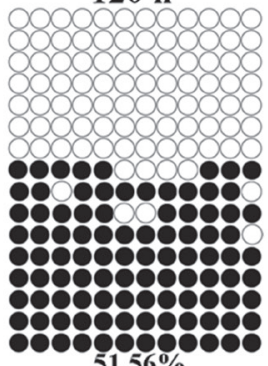

$\mathbf{5 1 . 5 6 \%}$

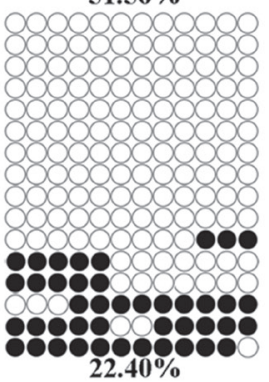

$22.40 \%$

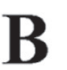

Aza(-)

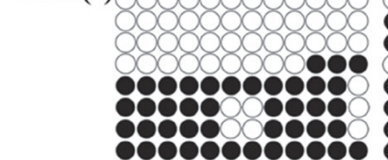

$\mathbf{2 2 . 9 2 \%}$

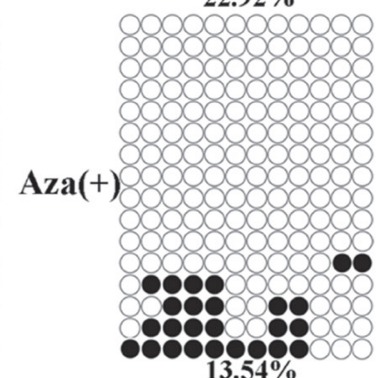

$13.54 \%$

4-cell

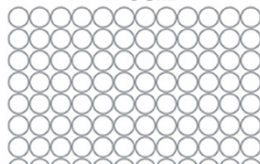

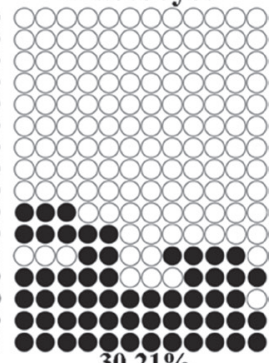

$30.21 \%$

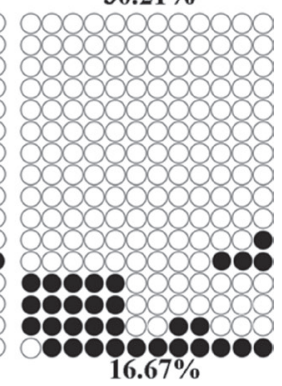

$D$
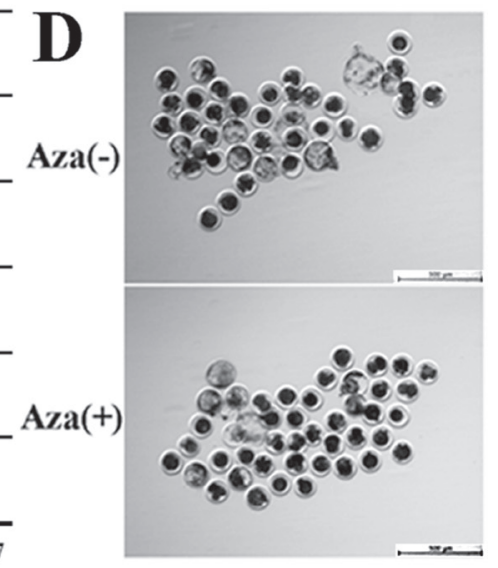

Figure 3: H19/Igf2 imprinting methylation statuses and transcription and the expression of genes regulating imprinting methylation in PFFs and cloned embryos and the cloned blastocysts derived from PFFs treated with 5-aza-dC. (A) the methylation statuses of H19/Igf2 imprinting in PFFs treated with 5-aza-dC, (B) the methylation statuses of H19/Igf2 imprinting at the 4-cell and blastocyst stages of cloned embryos derived from PFFs treated with 5-aza-dC, (C) the transcription of H19/Igf2 and genes regulating imprinting methylation at the 4-cell and blastocyst stages of cloned embryos derived from PFFs treated with 5-aza-dC, (D) the cloned blastocysts derived from PFFs treated with 5-aza-dC (Scale bar $=500 \mu \mathrm{m}$ ). Treating donor cells with 5-aza-dC resulted in the hypomethylated H19/Igf2 imprinting in PFFs and cloned embryos, disrupted H19 transcription, reduced expression of Igf2 and genes regulating imprinting methylation and downregulated blastocyst rate. Aza $(+)$ and Aza $(-)$ represented PFFs treated with 5-aza-dC or not. Black or white circles indicate methylated or unmethylated $\mathrm{CpG}$ sites, respectively. ${ }^{\mathrm{a}-\mathrm{b}}$ Values for a given gene with different superscripts differ significantly $(P<0.05)$. 
Table 3: Development of cloned embryos derived from the abnormal imprinting donor cells with H19 knockdown

\begin{tabular}{lccc}
\hline Group & No. embryos (Rep.) & No. embryos cleaved $(\% \pm$ SEM) & No. blastocysts $(\% \pm$ SEM) \\
\hline siRNA-control & $171(5)$ & $135(78.82 \pm 1.91)^{\mathrm{a}}$ & $22(12.85 \pm 1.37)^{\mathrm{a}}$ \\
siRNA-negative & $184(5)$ & $146(79.32 \pm 1.73)^{\mathrm{a}}$ & $21(11.34 \pm 1.28)^{\mathrm{a}}$ \\
siRNA-positive & $173(5)$ & $151(87.28 \pm 1.85)^{\mathrm{b}}$ & $48(27.90 \pm 1.84)^{\mathrm{b}}$ \\
\hline
\end{tabular}

${ }^{a-b}$ Values in the same column with different superscripts differ significantly $(P<0.05)$.

support the view that imprinting defect leads to the poor cloned embryo development [19]. It is known that imprinting can be faithfully retained by the maintenance enzymes including Dnmt1, Zfp57 and Trim28, etc [6]. Here, our study displayed that the significantly reduced expression of Dnmt1 and Zfp57 occurred in PFFs derived from the morphologically abnormal cloned fetuses, suggesting that the key molecules for genomic imprinting methylation maintenance was lost during the cloned embryo development, thereby resulting in the imprinting hypomethylation and the upregulated H19 transcription, further leading to the retarded cloned fetuses. Moreover, increasing studies also display that the retarded development of cloned fetuses is due to the aberrant imprinting $[4,14]$. Accordingly, the perturbed imprinting could be the cause of the morphological abnormality of cloned fetuses.

Previous studies have demonstrated that imprinting disruption is the cause of parthenogenetic or androgenetic embryo developmental failure, revealing that gametic imprinting is indispensable for the normal embryo development [11, 21]. Here, when PFFs with the abnormal imprinting were used as donor cells, along with the decreased expression levels of the imprinting methylation maintenance genes, the imprinting status was severely disrupted in cloned embryos, probably leading to the reduced development of cloned embryos. And, no significant differences of the imprinting status and embryo development were observed between the normal imprinting group and the control group, further suggesting that the error information in donor cells could be inherited to the cloned embryos and impair the embryo development.

Encouragingly, the regulation of genomic imprinting can rescue the failed development of parthenogenetic and semi-cloned embryos [22, 23], then, alteration of donor cell imprinting can further identity the concrete relationship between the donor cell imprinting status and the cloned embryo development. When donor cell imprinting methylation level was reduced by 5 -aza-dC, the hypomethylated imprinting occurred in cloned embryos and the blastocyst rate was significantly downregulated, further supporting the previous reports that treating donor cell with 5-aza-dC cannot enhance the cloned embryo development [24, 25]. H19 is a key regulator of the imprinted gene network [26]. The hypomethylated imprinting of H19/Igf2 leads to the high H19 transcription, and $\mathrm{H} 19$ biallelic expression has been reported in cloned embryos [14], then, it is speculated that the upregulated H19 expression in donor cells can be the cause of the low cloning efficiency, as the upregulated H19 transcription occurred in the abnormal imprinting group and the 5-aza$\mathrm{dC}$ treatment group. Expectedly, our results demonstrated that H19 knockdown in the abnormal imprinting group partly rescued the disrupted imprinting and enhanced the cloned embryo development. These improvements may be due to that H19 could interact with genes responsible for the imprinting methylation establishment and maintenance, and the relatively normal expression of Dnmt1, Zfp57 and Dnmt3a in cloned embryos can help explain this view. Certainly, numerous molecules can regulate genomic imprinting, and, more information is needed to clarify the imprinting regulatory mechanism during the cloned embryo development [8]. Taken together, donor cell imprinting status is critical for the cloned embryo development.

Indeed, the low development of cloned embryos is associated with the imprinting disruption, and the imprinting status in the individual embryo at the same stage, even if it can develop to the blastocyst or fetus, could be not all the same, suggesting that the imprinting status could also be different among the donor cells. In this study, only the imprinting status of the grouped fibroblasts not the real donor cells was examined to reveal the role of donor cell imprinting in the cloned embryo development, as it is impossible to detect the imprinting status in the individual donor cell that gives rise to its corresponding cloned embryo, then, the culture of cell colony derived from one single somatic cell and single cell bisulfite sequencing will be adopted to examine the precise role of donor cell imprinting regulating the cloned embryo development in the further study [27]. The imprinting data of cloned embryos was also based on dozens of pooled embryos, and whether imprinting is maintained or erased then reestablished in the normal cloned embryo development is still unclear, thus, embryo biopsy and single cell methylation and RNA sequencing will be further applied to reveal the real imprinting methylation status dynamics and the clear molecule regulatory mechanism of donor cell imprinting regulating SCNT mediated reprogramming $[28,29]$.

In conclusion, our results demonstrated that the imprinting status in PFFs derived from the morphologically abnormal cloned fetuses was aberrant, 

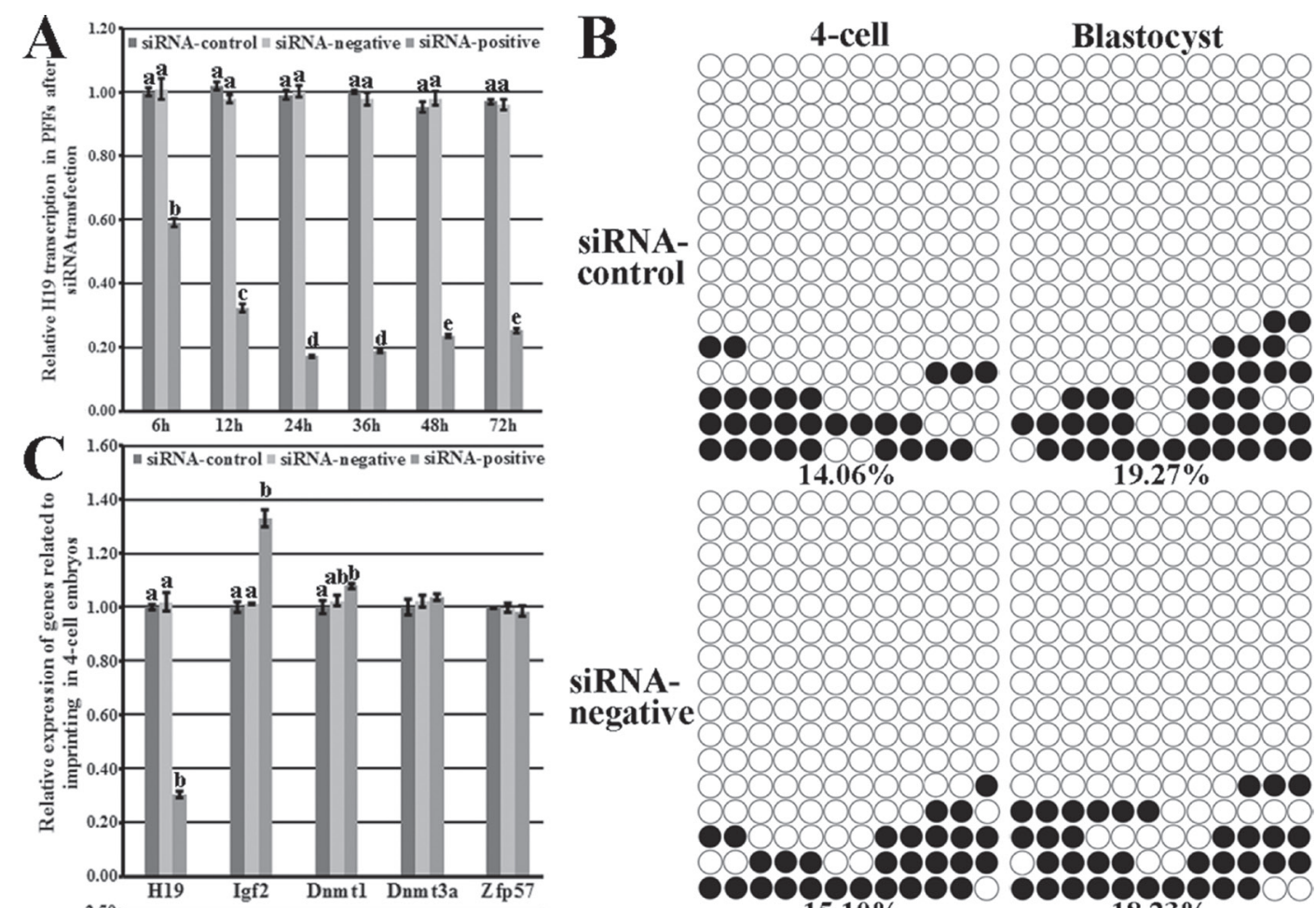

SiRNA-

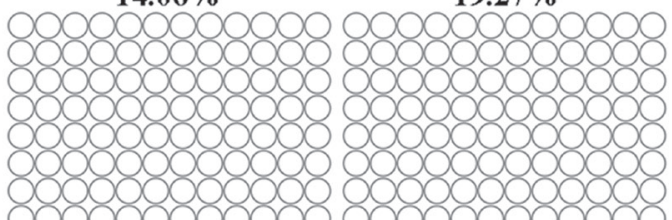

negative

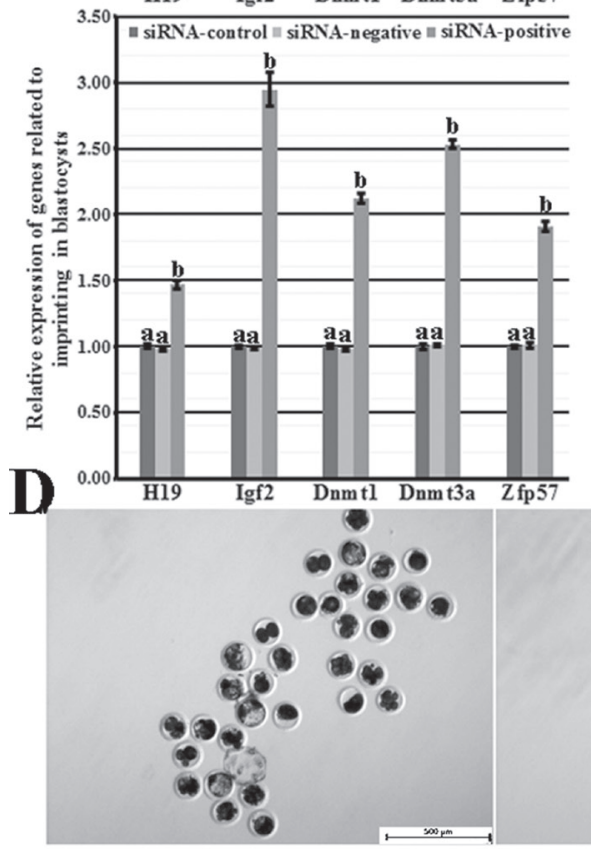

siRNA-control
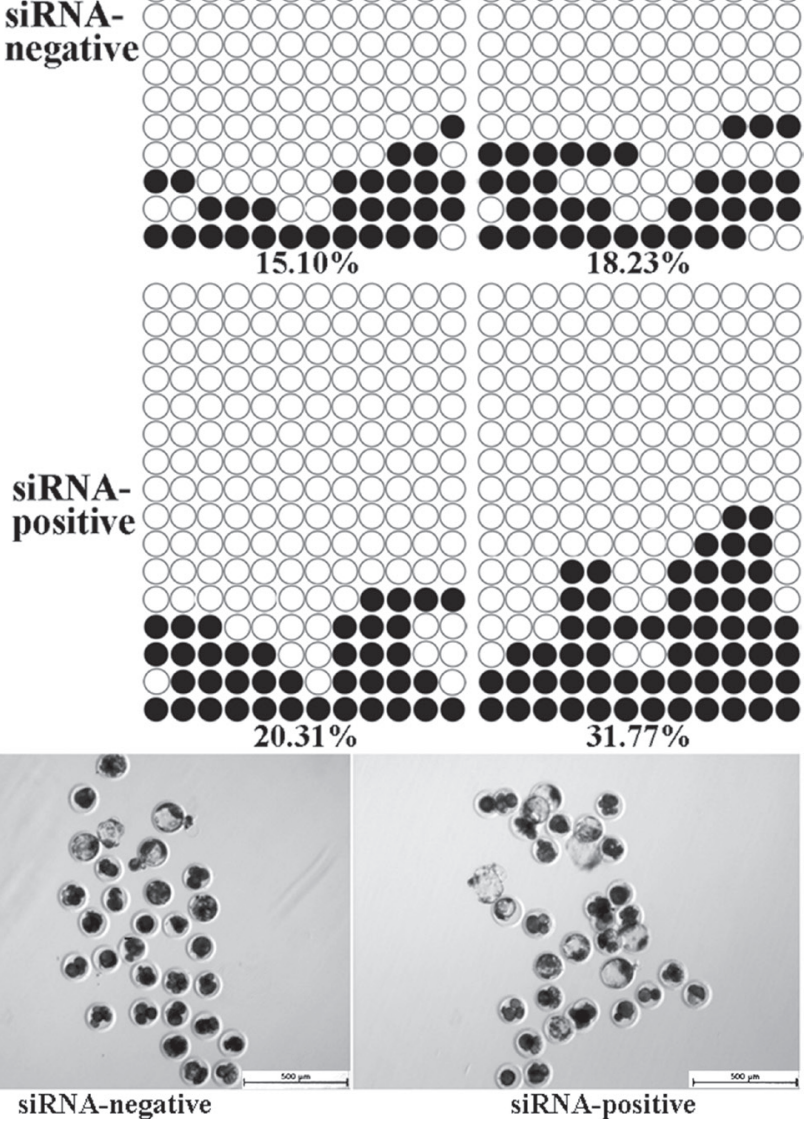

Figure 4: H19/Igf2 imprinting transcription and methylation statuses and the expression of genes regulating imprinting methylation in PFFs and cloned embryos, and the cloned blastocysts derived from the abnormal imprinting PFFs with H19 knockdown. (A) H19 transcription in the abnormal imprinting PFFs after siRNA transfection, (B) the methylation statuses of H19/ Igf2 imprinting at the 4-cell and blastocyst stages of cloned embryos derived from the abnormal imprinting PFFs in the siRNA-control, siRNA-negative and siRNA-positive groups, (C) the transcription of H19/Igf2 and genes regulating imprinting methylation at the 4-cell and blastocyst stages of cloned embryos derived from the abnormal imprinting PFFs in the siRNA-control, siRNA-negative and siRNApositive groups, (D) the cloned blastocysts derived from the abnormal imprinting PFFs in the siRNA-control, siRNA-negative and siRNApositive groups (Scale $b a r=500 \mu \mathrm{m}$ ). H19 knockdown in the abnormal imprinting PFFs improved H19/Igf2 imprinting methylation and transcription, the expression of genes regulating imprinting methylation and blastocyst rate. siRNA-control, siRNA-negative and siRNApositive represented the abnormal imprinting PFFs transfected with none siRNA, negative siRNA and positive siRNA, respectively. Black or white circles indicate methylated or unmethylated $\mathrm{CpG}$ sites, respectively. ${ }^{\mathrm{a}-\mathrm{c}}$ Values for a given gene with different superscripts differ significantly $(P<0.05)$. 
and these PFFs led to the even severely disrupted imprinting and reduced cloned embryo development. And, donor cell imprinting hypomethylation induced by 5 -aza$\mathrm{dC}$ was detrimental to the cloned embryo development, while H19 knockdown in the hypomethylated imprinting donor cells enhanced the cloned embryo development. These investigations suggest that donor cell imprinting can regulate the cloned embryo development and provide a new insight into improving the cloning efficiency and the health of cloned animals.

\section{MATERIALS AND METHODS}

Chemicals were purchased from Sigma-Aldrich Corporation (St. Louis, MO, USA), and disposable and sterile plasticware was obtained from Nunclon (Roskilde, Denmark), unless otherwise stated. All experiments were approved by the Animal Care Commission of Qingdao Agricultural University according to animal welfare laws, guidelines and policies. All surgery was performed under sodium pentobarbital anaesthesia, and all efforts were made to minimize suffering.

\section{Donor cell culture}

Donor cell culture has been described previously [25]. Briefly, porcine in vivo fertilized or cloned fetuses were obtained from sows after anaesthetized and sacrificed at day 35 of pregnancy, then PFFs were isolated from the fetuses under sodium pentobarbital anaesthesia. After the removal of fetal head, internal organs and limbs, the remaining tissues were finely minced into pieces, digested with $0.25 \%$ trypsin- $0.04 \%$ ethylenediaminetetraacetic acid solution (GIBCO), and dispersed in high glucose enriched Dulbecco's modified Eagle's medium (DMEM, GIBCO) containing 10\% fetal bovine serum (FBS, GIBCO) and $1 \%$ penicillin-streptomycin (GIBCO). The dispersed cells were centrifuged, resuspended and cultured in DMEM. Until confluence, PFFs were digested, centrifuged, resuspended in FBS containing 10\% dimethyl sulfoxide and stored in liquid nitrogen until use. Prior to SCNT, PFFs were thawed, cultured and used in 2-3 passages.

\section{Donor cell treatment}

For 5-aza-dC treatment [25], PFFs were cultured in DMEM supplemented with $10 \mathrm{nM}$ (the optimal concentration) 5-aza-dC without any antibiotics for $72 \mathrm{~h}$, $96 \mathrm{~h}$ or $120 \mathrm{~h}$, respectively.

For H19 knockdown, methods of siRNA design, synthesis and transfection have been reported in our study [3]. According to the requirement of Invitrogen Block-iT RNAi Designer and H19 mRNA information, the stealth siRNA was designed and synthesized, and the sequence was CCTCCTAGCTCTGACTCAAGAATAT. The negative sequence was CCTTAGCTCTGACTCAAGAACCTAT. Then, siRNAs were dissolved with Rnase free $\mathrm{H}_{2} \mathrm{O}$ to the concentration of $20 \mu \mathrm{M}$. Before transfection, PFFs were cultured in $400 \mu \mathrm{l}$ Opti-MEM (GIBCO), $1.5 \mu \mathrm{l}$ Lipofectamine 2000 (Lipo 2000, Invitrogen) was added into $50 \mu \mathrm{l} \mathrm{Opti-MEM}$ and incubated at room temperature for $5 \mathrm{~min}, 20 \mu \mathrm{M}$ H19 siRNA was diluted into $500 \mathrm{nM}$ with Opti-MEM, and, $100 \mu 1$ siRNA-Lipo 2000 complexes was obtained through a mixture of $50 \mu \mathrm{l}$ Opti-MEM with $500 \mathrm{nM}$ siRNA and $50 \mu \mathrm{l}$ Opti-MEM with $1.5 \mu \mathrm{l}$ Lipo 2000, incubated at room temperature for $30 \mathrm{~min}$ and added into each 24-well culture plate with PFFs and $400 \mu 1$ OptiMEM. After $6 \mathrm{~h}$, the medium including siRNA-Lipo 2000 complexes was replaced by DMEM containing 10\% FBS and $1 \%$ penicillin-streptomycin. The interference efficiency was examined at 6 h, 12 h, 24 h, 36 h, 48 h or $72 \mathrm{~h}$ posttransfection, respectively. The negative siRNA with the same amount was transfected as a control.

After 5-aza-dC treatment or H19 siRNA transfection, PFFs were harvested at $6 \mathrm{~h}, 12 \mathrm{~h}, 24 \mathrm{~h}$, $36 \mathrm{~h}, 48 \mathrm{~h}, 72 \mathrm{~h}, 96 \mathrm{~h}$ or $120 \mathrm{~h}$, respectively, and the cell number at every time point was determined with a hemacytometer. Then, the manner of 5-aza-dC treatment with the obvious imprinting hypomethylation or siRNA transfection with H19 significant knockdown but no notable effect on cell proliferation was applied in the subsequent experiment.

\section{Oocyte in vitro maturation}

Oocyte maturation has been reported [30]. Briefly, porcine ovaries were collected from a local slaughterhouse. Just after exposure, ovaries were placed into physiological saline with antibiotics at $37^{\circ} \mathrm{C}$ and transported to the laboratory. Follicles were aspirated, and follicular contents were washed with HEPES-buffered Tyrode's lactate. Cumulus-oocyte complexes (COCs) were recovered and cultured in maturation medium. After $42 \mathrm{~h}$, COCs were vortexed in hyaluronidase for $30 \mathrm{sec}$ to remove cumulus cells. Only oocytes with the visible polar body, regular morphology and homogenous cytoplasm were used.

\section{SCNT, embryo development and collection}

The procedure for SCNT has been described in our reports $[25,31]$. Briefly, matured oocytes and donor cells were placed into manipulation medium. After oocyte enucleation, donor cells were placed into the perivitelline space. Fusion and activation of the cell-cytoplast complexes were induced by electroporation. Then, the reconstructed embryos were cultured in porcine zygote medium-3 (PZM-3) for the subsequent development, and the cleavage and blastocyst rates were evaluated at $48 \mathrm{~h}$ and $156 \mathrm{~h}$ postactivation, respectively. For the collection 
of cloned embryos, 4-cell and blastocyst embryos in each group were collected at $48 \mathrm{~h}$ and $156 \mathrm{~h}$, respectively.

\section{Nuclear staining}

For blastocyst cell number, cloned embryos at $156 \mathrm{~h}$ postactivation were treated with acidic Tyrode's solution to remove zona pellucida, fixed in $4 \%$ paraformaldehyde for $30 \mathrm{~min}$, and stained with $10 \mu \mathrm{g} / \mathrm{ml}$ Hoechst 33342 for $5 \mathrm{~min}$ in the dark. After staining, cloned blastocysts were washed and mounted on slides. Then, blastocyst cell number was examined under ultraviolet light from a fluorescence microscope.

\section{Quantitative real-time PCR}

Measurement of gene expression with quantitative real-time PCR has been applied in our studies [30, 32]. Briefly, total RNA was extracted from $10^{4}$ PFFs or 50 pooled embryos at each stage using an RNeasy Micro Kit (Qiagen) according to the manufacturer's instructions, and the elution volume was $50 \mu$ l. Reverse transcription was performed using a PrimeScript RT Reagent Kit (TaKaRa). The $100 \mu \mathrm{l}$ reaction volume contained $20 \mu \mathrm{l}$ $5 \times$ PrimeScript Buffer, $5 \mu 1$ PrimeScript RT Enzyme Mix I, $5 \mu \mathrm{l}$ Oligo dT Primer $(50 \mu \mathrm{M}), 5 \mu \mathrm{l}$ Random 6 mers $(100 \mu \mathrm{M}), 50 \mu \mathrm{l}$ Total RNA and $15 \mu 1$ RNase Free $\mathrm{dH}_{2} \mathrm{O}$. The reaction condition was $37^{\circ} \mathrm{C}$ for $15 \mathrm{~min}$ and $85^{\circ} \mathrm{C}$ for $5 \mathrm{sec}$, and the cDNA was stored at $-20^{\circ} \mathrm{C}$ until use. For quantitative real-time $\mathrm{PCR}$, reactions were performed in 96-well optical reaction plates (Applied Biosystems) using SYBR Premix ExTaq II (TaKaRa) and a 7500 Real-Time PCR System (Applied Biosystems). Each reaction mixture $(20 \mu \mathrm{l})$ contained $2 \mu \mathrm{l}$ cDNA solution, $10 \mu 12 \times$ SYBR Premix Ex Taq II, $1.6 \mu \mathrm{l}$ PCR primers $(10 \mu \mathrm{M}), 0.4 \mu \mathrm{l}$ ROX Reference Dye II (50×) and $6 \mu 1 \mathrm{dH}_{2} \mathrm{O}$. Thermal cycling conditions were $95^{\circ} \mathrm{C}$ for $30 \mathrm{sec}, 40$ two-step cycles of $95^{\circ} \mathrm{C}$ for $5 \mathrm{sec}$ and $60^{\circ} \mathrm{C}$ for $34 \mathrm{sec}$, and finally a dissociation stage consisting of $95^{\circ} \mathrm{C}$ for $15 \mathrm{sec}, 60^{\circ} \mathrm{C}$ for $1 \mathrm{~min}$ and $95^{\circ} \mathrm{C}$ for $15 \mathrm{sec}$. For each sample, the cycle threshold (CT) values were obtained from three replicates. The primers used for the amplification of target and internal reference genes were presented in Supplementary Table 1. The relative expression levels of target genes were analyzed using the $2^{-\Delta \Delta \mathrm{CT}}$ method.

\section{Bisulfite sequencing}

Bisulfite sequencing has been reported [33]. Briefly, pooled samples were treated with sodium bisulfite to convert all unmethylated cytosine to uracil using an EZ DNA Methylation-Direct ${ }^{\mathrm{TM}}$ Kit (Zymo Research) according to the manufacturer's protocol. For PFFs, a Universal Genomic DNA Extraction Kit (TaKaRa) was used to extract genomic DNA. For samples of 50 4-cell or 10 blastocyst stage pooled zona pellucida-removed cloned embryos in each group, digestion was performed in M-Digestion Buffer plus with Proteinase $\mathrm{K}$ at $50^{\circ} \mathrm{C}$ for $20 \mathrm{~min}$. After treatment, a cytosine to thymine conversion was carried out at $98^{\circ} \mathrm{C}$ for $10 \mathrm{~min}$ and $64^{\circ} \mathrm{C}$ for $2.5 \mathrm{~h}$. Then, the samples were desalted, purified and diluted with M-Elution Buffer. Subsequently, nested PCR was carried out to amplify DMR3 of H19/Igf2 using the previously reported primers as described in Supplementary Table 2 and Hot Start Taq ${ }^{\mathrm{TM}}$ Polymerase (TaKaRa) with a profile of $94^{\circ} \mathrm{C}$ for $5 \mathrm{~min}, 40 \mathrm{cycles}$ of $94^{\circ} \mathrm{C}$ for $30 \mathrm{sec}, 55^{\circ} \mathrm{C}$ for $30 \mathrm{sec}$ and $72^{\circ} \mathrm{C}$ for $1 \mathrm{~min}$, followed by $72^{\circ} \mathrm{C}$ for $10 \mathrm{~min}$. Products from the first amplification reaction were used in the second PCR reaction, and the optimal annealing temperature of inner primers was $50^{\circ} \mathrm{C}$. Then, the amplified products were verified by electrophoresis and purified using an Agarose Gel DNA Purification Kit (TaKaRa), and the purified fragments were cloned into a pMD18-T Vector (TaKaRa) and subjected to sequence analysis.

\section{Statistical analysis}

Differences in data (mean \pm SEM) were analyzed with the SPSS statistical software. Statistical analyses of data concerning embryo development, gene expression and cell proliferation were performed with one-way ANOVA or $t$-test when two groups were compared. For all analyses, differences were considered to be statistically significant when $P<0.05$.

\section{Abbreviations}

SCNT, somatic cell nuclear transfer; DMR, differentially methylated region; ICR, imprinting control region; PFFs, porcine fetal fibroblasts; FBS, fetal bovine serum; DMEM, Dulbecco's modified Eagle's medium; COCs, cumulus-oocyte complexes; CT, cycle threshold; PZM-3, porcine zygote medium-3.

\section{Author contributions}

Yanjun Huan conceived the project and wrote the manuscript. Xuexiong Song, Fangzheng Li and Zhongling Jiang supervised the experiment, performed the molecular analysis and revised the manuscript. Yueping Sun, Huatao Li, Shansong Gao, Liping Zhang and Guimin Zhao produced the cloned embryos. Binghua Xue and Jingyu Li collected PFFs. Hongbin He and Zhonghua Liu participated in data analysis.

\section{ACKNOWLEDGMENTS}

We are thankful to Doctor Lingling Song in Shandong Academy of Agricultural Sciences and Doctor Jianyu Wang in Northeast Agricultural University for their helpful discussion. 


\section{CONFLICTS OF INTEREST}

The authors declare that no conflicting financial interests exist.

\section{FUNDING}

This work was supported by grants from National Natural Science Foundation of China, NSFC (31602019 to HYJ), High-level Talent Research Foundation of Qingdao Agricultural University (1116029 to HYJ) and Natural Science Foundation of Shandong Province (BS2015SW014 to HYJ).

\section{REFERENCES}

1. Lee K, Prather RS. Advancements in somatic cell nuclear transfer and future perspectives. Animal Frontiers. 2013; 3:56-61.

2. Huili J, Haosheng L, Dengke P. Epigenetic reprogramming by somatic cell nuclear transfer: questions and potential solutions. Yi Chuan. 2014; 36:1211-1218.

3. Song XX, Liu ZH, He HB, Wang JY, Li HT, Li JY, Li FZ, Jiang ZL, Huan YJ. Dnmt1s in donor cells is a barrier to SCNT-mediated DNA methylation reprogramming in pigs. Oncotarget. 2017; 8:34980-34991. https://doi.org/10.18632/ oncotarget. 16507.

4. Huan YJ, Zhu J, Huang B, Mu YS, Kong QR, Liu ZH. Trichostatin A rescues the disrupted imprinting induced by somatic cell nuclear transfer in pigs. PLoS One. 2015; 10:e0126607.

5. Barlow DP, Bartolomei MS. Genomic imprinting in mammals. CSH Perspect Biol. 2014; 6:a018382.

6. Messerschmidt DM. Should I stay or should I go Protection and maintenance of DNA methylation at imprinted genes. Epigenetics. 2012; 7:969-975.

7. Okae H, Matoba S, Nagashima T, Mizutani E, Inoue K, Ogonuki N, Chiba H, Funayama R, Tanaka S, Yaegashi N, Nakayama K, Sasaki H, Ogura A, et al. RNA sequencingbased identification of aberrant imprinting in cloned mice. Hum Mol Genet. 2014; 23:992-1001.

8. Bartolomei MS, Ferguson-Smith AC. Mammalian genomic imprinting. CSH Perspect Biol. 2011; 3:a002592.

9. Li B, Chen SQ, Tang N, Xiao XF, Huang JL, Jiang F, Huang XY, Sun FZ, Wang XH. Assisted reproduction causes reduced fetal growth associated with downregulation of paternally expressed imprinted genes that enhance fetal growth in mice. Biol Reprod. 2016; 94:1-11.

10. Zhong C, Yin Q, Xie Z, Bai M, Dong R, Tang W, Xing YH, Zhang H, Yang S, Chen LL, Bartolomei MS, Ferguson Smith A, Li D, et al. CRISPR-Cas9-mediated genetic screening in mice with haploid embryonic stem cells carrying a guide RNA library. Cell Stem Cell. 2015; 17:221-232.

11. Hu M, TuanMu LC, Wei H, Gao F, Li L, Zhang S. Development and imprinted gene expression in uniparental preimplantation mouse embryos in vitro. Mol Biol Rep. $2015 ; 42: 345-353$.

12. Blelloch R, Wang Z, Meissner A, Pollard S, Smith A, Jaenisch R. Reprogramming efficiency following somatic cell nuclear transfer is influenced by the differentiation and methylation state of the donor nucleus. Stem Cells. 2006; 24:2007-2013.

13. Liu J, Wang Y, Su J, Luo Y, Quan F, Zhang Y. Nuclear donor cell lines considerably influence cloning efficiency and the incidence of large offspring syndrome in bovine somatic cell nuclear transfer. Reprod Domest Anim. 2013; 48: 660-664.

14. Suzuki J, Therrien J, Filion F, Lefebvre R, Goff AK, Perecin F, Meirelles FV, Smith LC. Loss of methylation at H19 DMD is associated with biallelic expression and reduced development in cattle derived by somatic cell nuclear transfer. Biol Reprod. 2011; 84:947-956.

15. Shen CJ, Lin CC, Shen PC, Cheng WT, Chen HL, Chang TC, Liu SS, Chen CM. Imprinted genes and satellite loci are differentially methylated in bovine somatic cell nuclear transfer clones. Cell Reprogram. 2013; 15:413-424.

16. Xu WH, Li ZC, Yu B, He XY, Shi JS, Zhou R, Liu DW, $\mathrm{Wu} \mathrm{ZF}$. Effects of DNMT1 and HDAC inhibitors on genespecific methylation reprogramming during porcine somatic cell nuclear transfer. PLoS One. 2013; 8:e64705.

17. Zhang XY, Wang DX, Han Y, Duan FF, Lv QY, Li ZJ. Altered imprinted gene expression and methylation patterns in mid-gestation aborted cloned porcine fetuses and placentas. J Assist Reprod Genet. 2014; 31:1511-1517.

18. Huan YJ, Wu ZF, Zhang JG, Zhu J, Xie BT, Wang JY, Li JY, Xue BH, Kong QR, Liu ZH. Alteration of the DNA methylation status of donor cells impairs the developmental competence of porcine cloned embryos. J Reprod Dev. 2016; 62:71-77.

19. Tian X. Genomic Imprinting in farm animals. Annu Rev Anim Biosci. 2014; 2:23-40.

20. Wan YJ, Deng MT, Zhang GM, Ren CF, Zhang H, Zhang YL, Wang LZ, Wang F. Abnormal expression of DNA methyltransferases and genomic imprinting in cloned goat fibroblasts. Cell Biol Int. 2016; 40:74-82.

21. Reik W, Walter J. Genomic imprinting: parental influence on the genome. Nat Rev Genet. 2001; 2:21-32.

22. Kono T, Obata Y, Wu Q, Niwa K, Ono Y, Yamamoto Y, Park ES, Seo JS, Ogawa H. Birth of parthenogenetic mice that can develop to adulthood. Nature. 2004; 428:860-864.

23. Zhong C, Li J. Efficient Generation of gene-modified mice by haploid embryonic stem cell-mediated semi-cloned technology. Methods Mol Biol. 2017; 1498:121-133.

24. Enright BP, Sung LY, Chang CC, Yang X, Tian $\mathrm{XC}$. Methylation and acetylation characteristics of cloned bovine embryos from donor cells treated with 5-aza-2'-deoxycytidine. Biol Reprod. 2005; 72: 944-948.

25. Huan YJ, Zhu J, Xie BT, Wang JY, Liu SC, Zhou Y, Kong QR, He HB, Liu ZH. Treating Cloned Embryos, But 
Not Donor Cells, with 5-aza-2'-deoxycytidine Enhances the Developmental Competence of Porcine Cloned Embryos. J Reprod Dev. 2013; 59:442-449.

26. Gabory A, Ripoche MA, Le Digarcher A, Watrin F, Ziyyat A, Forne T, Jammes H, Ainscough JFX, Surani MA, Journot L, Dandolo L. H19 acts as a trans regulator of the imprinted gene network controlling growth in mice. Development. 2009; 136:3413-3421.

27. Smallwood SA, Lee HJ, Angermueller C, Krueger F, Saadeh H, Peet J, Andrews SR, Stegle O, Reik W, Kelsey G. Single-cell genome-wide bisulfite sequencing for assessing epigenetic heterogeneity. Nat Methods. 2014; 11:817-820.

28. Liu W, Liu X, Wang C, Gao Y, Gao R, Kou X, Zhao Y, Li J, Wu Y, Xiu W, Wang S, Yin J, Liu W, et al. Identification of key factors conquering developmental arrest of somatic cell cloned embryos by combining embryo biopsy and singlecell sequencing. Cell Discov. 2016; 2:16010.

29. Ling KY, Cheow LF, Quake SR, Burkholder WF, Messerschmidt DM. Single cell restriction enzyme-based analysis of methylation at genomic imprinted regions in preimplantation mouseeEmbryos. Methods Mol Biol. 2017; 1605:171-189.
30. Huan YJ, Xie BT, Liu SC, Kong QR, Liu ZH. A novel role for DNA methyltransferase 1 in regulating oocyte cytoplasmic maturation in pigs. PLoS One. 2015; 10:e0127512.

31. Huan YJ, Hu K, Xie BT, Shi YQ, Wang F, Zhou Y, Liu SC, Huang B, Zhu J, Liu ZF, He YL, Li JY, Kong QR, et al. Ovulation statuses of surrogate gilts are associated with the efficiency of excellent pig cloning. PLoS One. 2015; 10:e0142549.

32. Huan YJ, Wang HM, Wu ZF, Zhang JG, Liu ZH, He HB. The expression patterns of DNA methylation reprogramming related genes are associated with the developmental competence of cloned embryos after zygotic genome activation in pigs. Gene Expr Patterns. 2015; 18:1-7.

33. Huan YJ, Wu ZF, Zhang JG, Zhu J, Liu ZH, Song XX. Epigenetic modification agents improve gene-specific methylation reprogramming in porcine cloned embryos. PLoS One. 2015; 10:e129803. 